\title{
Actividad in vitro anti-Leishmania (Trypanosomatidae) del epóxido trans-Z-a-bisaboleno y del Safrol, en frutos de Piper auritum (Piperaceae)
}

\author{
Daniela Rodríguez-Chaves ${ }^{1}$, Vanessa Bagnarello-Madrigal ${ }^{1}$, Javier Alpizar-Cordero ${ }^{1}$, \\ Alonso Calvo-Vargas ${ }^{1}$, Maribel Cordero-Villalobos ${ }^{1}$, Misael Chinchilla-Carmona ${ }^{2}$, \\ Idalia Valerio-Campos ${ }^{2} \&$ Ronald Sánchez Porras ${ }^{3}$ \\ 1. Departamento de Química, Universidad de Ciencias Médicas (UCIMED), San José, Costa Rica; rodriguezcd@ \\ ucimed.com, bagnarellomv@ucimed.com, alpizarcj@ucimed.com, calvova@ucimed.com, corderovm@ucimed.com \\ 2. Departamento de Investigación Básica, Universidad de Ciencias Médicas (UCIMED), San José, Costa Rica; chin- \\ chillacm@ucimed.com, valerioci@ucimed.com \\ 3. Sección de Biología, Sede Occidente, Universidad de Costa Rica, San José, Costa Rica; rsanchez@ucr.ac.cr
}

Recibido 08-XII-2017. Corregido 09-III-2018. Aceptado 10-IV-2018.

\begin{abstract}
Anti-Leishmania (Trypanosomatidae) activity of trans-Z- $\alpha$-bisabolene epoxide and isolation of safrole, in fruits of Piper auritum (Piperaceae). The leishmaniosis disease incidence is high in tropical regions, and its current treatment has shown severe secondary effects. Considering this problem, many studies have focused on plants, looking for chemical components that have anti-leishmanial activity, and are free of adverse effects for human beings. The purpose of this work was to find a chemical component with this kind of activity in Piper auritum. In a phytochemical screening of this plant, we found some cumarins, terpens, triterpens and reducing sugars; and later, we identified the components trans-Z- $\alpha$-bisabolene epoxide and Safrol. The first component presented a $\mathrm{CI}_{50}$ of $50.0 \mu \mathrm{g} / \mathrm{mL}$ of anti-Leishmania activity. The Safrol, which is the major component of the essential oils of this plant, did not show antiparasitic activity. These results are discussed considering treatment of leishmaniasis. Rev. Biol. Trop. 66(2): 826-835. Epub 2018 June 01.
\end{abstract}

Key words: Leishmania; Leishmaniasis; Piper auritum; phytochemical screening; treatment.

El género Leishmania fue descubierto en el siglo XIX por William Boog. A través de los años se han identificado varias especies distribuidas por el mundo y son la leishmaniasis cutánea y visceral las principales patologías asociadas con este parásito (De Castro et al., 2015). A nivel mundial la leishmaniasis visceral es responsable de aproximadamente 59000 muertes, de las cuales 35000 pacientes son varones y 24000 son mujeres y se considera que la incidencia está en aumento, porque muchos de los casos no son reportados o son mal diagnosticados (WHO, 2016). La leishmaniasis visceral puede causar fiebre prolongada, anemia, hemorragias, entre otras sintomatologías más severas en las que, si no hay un tratamiento eficaz, los casos pueden llegar a ser mortales. Como en otros procesos infecciosos, si existe una enfermedad concomitante inmunosupresora, como es el caso de las infecciones por VIH, se generan mayores complicaciones que incluso inducen la reducción de la eficiencia del tratamiento retroviral (Hasnain, Ghosh, Baker, \& Mondal, 2014).

Los síntomas en los pacientes que padecen leishmaniasis cutánea dependen de la especie del parásito infectante y del estado inmunológico del individuo, estos presentan como característica común, lesiones ulceradas en la piel, a nivel de boca y tracto respiratorio superior, incluso con destrucción en el paciente partes de la nariz, faringe y laringe, en caso de no recibir un tratamiento adecuado (Bezerra, Barros, \& De Francesco, 2014). 
Factores externos como el cambio climático están asociados a futuras proliferaciones de la leishmaniasis cutánea, debido a la migración de diversas especies de reservorios; en Costa Rica se han presentado fluctuaciones de especies debidas principalmente al fenómeno del niño. Su incidencia ha aumentado de 10.5 casos por cada 100000 habitantes en 2001, a 41.6 en 2007 por cada 100000 habitantes. Las especies más comunes halladas en Costa Rica son la $L$. panamensis y la L. braziliensis, las cuales causan la leishmaniasis cutánea y muco-cutánea (Jiménez, Rojas, Vargas, \& Herrero, 2000; Jaramillo-Antillón, Espinoza-Aguirre, \& LoboPhilp, 2009). En 1999 se reportó en Costa Rica el primer caso de leishmaniasis visceral, causado por la especie $L$. donovani infantum o L. donovani chagasi (Carrillo, Chinchilla, Valverde, Porras, \& Mora, 1999).

Las principales drogas contra estos parásitos se basan en compuestos antimoniales utilizados en altas concentraciones, que debido a la prolongada dosificación, producen efectos cardiotóxicos, hepatotóxicos y nefrotóxicos (Barbosa, 2001; Croft \& Coombs, 2003).

Muchas de las enfermedades parasitarias ocurren en países pobres que no tienen presupuesto para pagar los precios de los medicamentos, por lo que dado que el uso de plantas para el tratamiento de estas enfermedades es muy común, el estudio de nuevos compuestos activos aislados de plantas, es una alternativa que disminuiría el uso de las drogas sintéticas (Wink, 2012).

En un estudio previo, se reportó la actividad antiparasitaria de 67 plantas de la Reserva Biológica Alberto Manuel Brenes (ReBAMB), entre ellas la del extracto crudo de Piper auritum de la familia Piperaceae, que mostró resultados promisorios (Chinchilla-Carmona et al., 2014). Este género es, a nivel mundial, uno de los más estudiados, identificándose aproximadamente 2000 especies ubicadas entre la región tropical y subtropical. Sus especies son utilizadas desde hace varios siglos como remedios caseros debido a su actividad, antimicrobiana, anti-inflamatoria y anti-fúngica (Halkimi, Ahmad, Heng, \& Mohd, 2011). Se ha observado que la familia Piperaceae tiene acción insecticida, moluscicida, antimalárica, esto debido a los tipos de metabolitos secundarios presentes en cada género (Leyva et al., 2009), dentro de los que se destacan los lignanos, sesquiterpenos, diterpenos, monoterpenos y compuestos fenólicos (García, Leyva, Martínez, \& Stashenko, 2007).

Monzote, García, Montalvo, Scull y Miranda (2010) reportaron la actividad contra la leishmania del aceite esencial de Piper auritum, debido a que inhibió el crecimiento de promastigotes de todas las especies de Leishmania utilizadas en el estudio; se identificó también el grupo de metabolitos secundarios responsables de la actividad anti-parasitaria, sin especificar el(los) compuesto(s) químico(s), presentes en la planta al (los), que se le atribuye dicha actividad.

En este trabajo, se reporta la actividad antiparasitaria contra Leishmania sp. de los compuestos trans-Z-alfa-bisaboleno epóxido y el Safrol aislado de la planta $P$. auritum recolectada en una Reserva Biológica de Costa Rica.

\section{MATERIALES Y MÉTODOS}

Recolección del material: Se trabajó con los frutos inmaduros y maduros de la planta P. auritum, (número 72 en el herbario de la UCIMED), recolectados en la Reserva Biológica Alberto Manuel Brenes (REBAMB); ésta reserva se localiza a $42 \mathrm{~km}$ noreste de San Ramón, (10¹3’49” N - 84³6’10”), Alajuela, Costa Rica, cuya altitud varía entre los 600 y $1640 \mathrm{~m}$ de altura, con una temperatura promedio de $21{ }^{\circ} \mathrm{C}$ y una humedad relativa del $98 \%$. (Sánchez, 2000). El material fue recolectado el 26 de abril 2011, y fue trasladado al laboratorio de parasitología de la Universidad de Ciencias Médicas en una hielera a baja temperatura. $P$. auritum es una planta perenne por lo que los frutos se encuentran durante todo el año.

Preparación del extracto: A un total de $500 \mathrm{~g}$ de los frutos maduros y los inmaduros de la planta $P$. auritum que ya habían dado actividad antiparasitaria en estudios previos 
(Chinchilla-Carmona et al., 2014), fueron mezclados y secados en un horno a una temperatura de $40{ }^{\circ} \mathrm{C}$ durante una semana, y posteriormente molidos en un picador/triturador marca Trapp ${ }^{\circledR}$. El material vegetal se maceró con una disolución de etanol al $70 \% \mathrm{v} / \mathrm{v}$ en una botella ámbar por una semana, con agitación ocasional; los extractos fueron filtrados al vacío con papel Whatman 1 , luego se concentraron a $40{ }^{\circ} \mathrm{C}$ en un evaporador rotatorio (Büchi R-114, fabricado Suiza), obteniéndose el extracto crudo. Este extracto fue probado en el laboratorio de parasitología para medir su actividad. Para la obtención del $\mathrm{CI}_{50}$ fue necesaria la medición del peso seco de la muestra (Chinchilla-Carmona et al., 2011a, b).

Tamizaje fitoquímico: Posteriormente, se realizó el tamizaje fitoquímico del extracto según lo establecido por Sharapin (2000), con algunas modificaciones. El procedimiento fue el siguiente: $40 \mathrm{~mL}$ del extracto crudo se mezclaron con $10 \mathrm{~mL}$ de agua destilada; posteriormente, se realizaron tres extracciones líquido-líquido con éter etílico. El extracto etéreo obtenido se concentró a sequedad y se le denominó E. El extracto acuoso que se separó de la extracción líquido-líquido fue separado en dos recipientes diferentes; a una de las partes se le denominó AQ1, conservándola como tal, mientras que la otra parte fue hidrolizada con $10 \mathrm{~mL} \mathrm{HCl} 3 \mathrm{~mol} / \mathrm{L}$ durante $15 \mathrm{~min}$.

$\mathrm{Al}$ extracto hidrolizado se le volvió a realizar una extracción líquido-líquido con 3 x 10 $\mathrm{mL}$ de éter etílico, obteniéndose un segundo extracto etéreo (AQ2) y el extracto acuoso residual (AQ2-hidrolizado).

A cada uno de los extractos se les realizaron las pruebas cualitativas correspondientes para determinar los metabolitos secundarios presentes en cada parte del extracto, según lo mencionado por Sharapin (2000), a las fases etéreas (E y AQ2) se les aplicó las pruebas químicas para la determinación de: terpenos (Vainillina), alcaloides (Dragendorff), flavonoides (Shinoda), cumarinas $(\mathrm{KOH})$, triterpenos y esteroles (Lieberman-Burchard), quinonas (Bornträger-Kraus). A la muestra acuosa (AQ1) se les determinó la presencia de: fenoles-taninos $\left(\mathrm{FeCl}_{3}\right)$, polisacáridos (Lugol), azúcares reductores (Fehling), saponinas (espuma), y alcaloides (Dragendorff). A la muestra acuosa hidrolizada (AQ2-hidrolizada) se le determinó también la presencia de antocianinas por medio de una prueba ácido-base.

Muestras de los extractos E, AQ1 y AQ2 fueron preparadas para analizar su actividad contra Leishmania sp., para esto se pesó una cantidad conocida de la muestra y se disolvió en una disolución al $10 \%$ de DMSO, según lo menciona la literatura (Fernández et al., 2009).

El extracto que presentó actividad fue el etéreo por lo que algunos de los metabolitos presentes en este extracto es el responsable de la actividad antiparasitaria, donde se destaca la presencia de cumarinas, terpenos o esteroles.

Aislamiento del epóxido trans-Z-abisaboleno: Como la fase etérea (E) presentó actividad contra el parásito, se procedió a preparar más extracto crudo que consistió en secar, triturar y macerar nuevos frutos con una disolución hexano - éter etílico (9:1), durante 7 días con agitación ocasional, esto con el objetivo de extraer principalmente los metabolitos secundarios con carácter hidrofóbico. El extracto crudo se concentró en un evaporador rotatorio marca Büchi R-114 a $30{ }^{\circ} \mathrm{C}$, y se procedió a la separación de sus componentes mediante cromatografía de columna, se utilizó como fase estacionaria gel de sílice con un Mesh de 230-400, y como fase móvil se realizó un gradiente de concentración de los solventes hexano y acetato de etilo, se inició con un 100 $\%$ hexano y terminó con una mezcla 7:3 acetato de etilo:hexano, obteniéndose 57 fracciones de $10 \mathrm{~mL}$ cada una. A las fracciones recolectadas se les realizó una capa fina (TLC), se usó una placa de Si250F TLC, esto para poder ver el comportamiento de los metabolitos aislados, y se procedió a unir las fracciones que presentaran un comportamiento igual durante el revelado de las placas con UV, yodo y vainillina.

Las fracciones resultantes fueron preparadas según el procedimiento de disolución en DMSO al $10 \%$ para ser probada su actividad 
antiparasitaria. Las fracciones que resultaron tener actividad antiparasitaria fueron la P4 y P7.

Con estas muestras ya semipurificadas, se seleccionaron las señales de la capa fina que presentaron una mejor tinción con la vainillina, y se procedió a realizar una capa preparativa con la muestra, se utilizó una placa de 20 × 20 $\mathrm{cm}$ con las mismas características del TLC. Se revelaron las orillas de la placa y se rasparon las muestras que tenían un $\mathrm{Rf}$ igual al señalado; posteriormente, se extrajo el material de la sílica gel con metanol y se concentró el mismo hasta sequedad. Se repitió el procedimiento en capa preparativa hasta obtener una cantidad de muestra que alcanzara para realizar las pruebas parasitológicas finales, y poder identificar el metabolito por GC-MS (cromatografía de gases, acoplado a un espectrómetro de masas).

Análisis de la muestra por GC-MS: La identificación del compuesto aislado se realizó mediante cromatografía de gases, con equipo marca Shimadzu, modelo GC-17A, con detector de espectrómetro de masas, modo split; modelo QP-5000, acoplado con una columna AT-5 (5\% difenilpolisiloxano), longitud $30 \mathrm{~m}$, $0.25 \mathrm{~mm}$ ID y detector de ionización (impacto electrónico $70 \mathrm{eV}$ ), la base de datos del equipo (marca: Wiley139, NIST107 y SZTERP) identificó el metabolito como el trans-Z- $\alpha$ bisaboleno, el cual según la bibliografía, es un metabolito presente en la planta $P$. auritum (Hernández, Rodríguez, García, \& Pino, 2003).

Aislamiento del Safrol: Para el aislamiento del Safrol se trabajó con las fracciones separadas en la columna de la siguiente manera: hubo un comportamiento igual para la capa fina realizada a las fracciones P15 hasta P26, por lo que se procedió a unirlas, y como la capa fina presentó una única señal (solo una mancha fue revelada con vainillina, UV y yodo) se definió que el compuesto tenía una alta pureza, por lo que se procedió a enviar la fracción al CIPRONA en la UCR para la identificación del compuesto. Para esto se realizó el análisis por medio de GC-MS y la base de datos del equipo identificó al Safrol como único compuesto presente en esta fracción, esto se corroboró con artículos donde se menciona al Safrol como metabolito mayoritario del aceite esencial del P. auritum (Monzote et al., 2010); esto explicó que estuviese presente en tantas fracciones de la columna. Esta misma fracción fue enviada a parasitología para realizar los análisis correspondientes, y se determinó que el compuesto no presentaba actividad antiparasitaria.

Parásitos: Los promastigotos de la cepa OCR (suministrada por el Dr. Rodrigo Zeledón), de características ampliamente conocidas, fueron cultivados en medio de Rugai para el mantenimiento de la cepa, y específicamente para los experimentos, en medio RPMI suplementado con suero fetal bovino al $10 \%$.

Ensayos in vitro: De acuerdo con los métodos descritos previamente (ChinchillaCarmona et al. 2014), se probó la actividad antiparasitaria de los extractos en estudio, así como del componente final obtenido, mantenidos en congelación por no más de dos meses antes de su uso, de la siguiente manera. El material, a diferentes concentraciones, fue puesto en contacto durante $24 \mathrm{~h}$ en refrigeración, con $5 \times 10^{5}$ a $10^{6}$ promastigotos obtenidos de un cultivo en fase logarítmica, Después de este periodo de tiempo, la viabilidad de los parásitos fue determinada por medio del azul tripano (Trypan blue), la lectura se hizo en no más de dos minutos después del montaje de la muestra. Cada proceso se hizo por triplicado, en no menos de tres experimentos sucesivos diferentes, y la lectura fue realizada por una sola persona, uno de los coautores, en todos los casos. Las diferentes concentraciones de los extractos o del producto purificado sirvieron para determinar la concentración inhibitoria del $50 \%$ de los organismos $\left(\mathrm{CI}_{50}\right)$. El método de Probit (Díaz, Bulus, \& Pica, 2004) fue usado especialmente para el cálculo de $\mathrm{CI}_{50}$ de la actividad antiparasitaria encontrada. Este método, toma los diferentes datos obtenidos para cada dilución del extracto, con peso conocido del componente en estudio, en cuanto a su actividad antiparasitaria, menor y mayor al $50 \%$; 
con esta información, se establece el gráfico respectivo en que se comparan efecto vs. dosis, aspecto fundamental en el cálculo de $\mathrm{CI}_{50}$. Los controles negativos, parásitos en medio MEM únicamente y positivos, parásitos muertos por calentamiento, fueron adicionados en todos los experimentos.

Pruebas de toxicidad: Para el estudio del efecto tóxico de los productos se siguieron los procedimientos previamente establecidos (Luize et al., 2005, Chinchilla-Carmona et al., 2014), se utilizó como modelo la lisis eritrocítica. Para ello se extrajo sangre en tubos heparinizados, de ratones blancos, hembras o machos (cepa Swiss Hds: CD-1) con un peso promedio de $25 \mathrm{~g}$; esta sangre se colocó en contacto con diferentes diluciones de los extractos, crudos o semi-purificados por $24 \mathrm{~h}$ en refrigeración. La toxicidad fue determinada por la observación de lisis o aglutinación globular, se estableció la dilución 1:80 como parámetro límite de toxicidad; todas estas pruebas se realizaron en triplicado. Todo el manejo de los animales se realizó con la ética y los permisos correspondientes (CICUA-07-10).

\section{RESULTADOS}

El tamizaje fitoquímico realizado al extracto hidroalcohólico de una muestra que contenía los frutos maduros e inmaduros, correspondientes a la planta $P$. auritum (Cuadro 1), evidencia la presencia de cumarinas, terpenos, triterpenos y/o esteroles y azúcares reductores.

Después del correspondiente proceso de purificación, se lograron aislar dos componentes de los frutos; el trans-Z-alfa-bisaboleno (A) y el Safrol (B) (Fig. 1).

El espectro de masas del trans-Z-alfabisaboleno se muestra en la figura 2; este compuesto presentó actividad contra Leishmania sp. con un valor de $\mathrm{CI}_{50}$ de $50.00 \mu \mathrm{g} / \mathrm{mL}$. El Safrol fue aislado e identificado por la base de datos del equipo, este compuesto no presentó actividad antiparasitaria contra Leishmania sp.

\section{DISCUSIÓN}

La leishmaniasis es una enfermedad causada por protozoos del género Leishmania (Hernández-Torres et al., 2015); la infección se transmite por medio de la picadura de moscos

\section{CUADRO 1}

Tamizaje fitoquímico de los frutos inmaduros y maduros de la planta Piper auritum

TABLE 1

Phytochemical screening of immature and mature fruits of the Piper auritum plant

\begin{tabular}{|c|c|c|c|}
\hline Metabolito secundario & Extracto etéreo & Extracto acuoso & Extracto etéreo de la fase acuosa hidrolizada \\
\hline Alcaloide & - & - & - \\
\hline Flavonoide & - & - & - \\
\hline Cumarina & + & - & + \\
\hline Triterpenos y esteroles & + & & + \\
\hline Quinonas & - & - & - \\
\hline Taninos & - & - & \\
\hline Azúcares reductores & & + & \\
\hline Antocianinas & & & - \\
\hline Terpenos & + & & \\
\hline$\underline{\text { Saponinas }}$ & - & - & \\
\hline
\end{tabular}

Nota: + Presencia del metabolito secundario, - Ausencia del metabolito secundario 


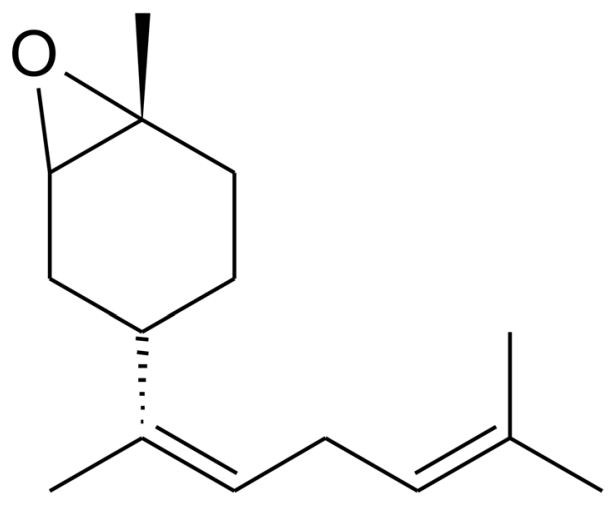

A
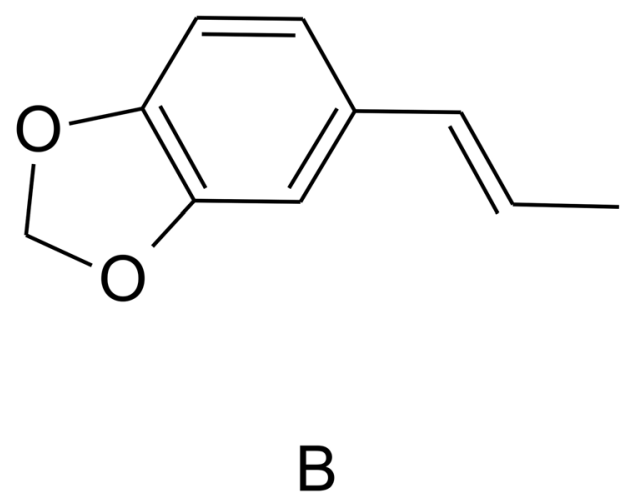

Fig. 1. Estructuras químicas del epóxido trans-Z- $\alpha$-bisaboleno (A) y del Safrol (B) aislados del fruto inmaduro y maduro de $P$. auritum.

Fig. 1. Chemical structures of the trans-Z- $\alpha$-bisabolene epoxide (A) and Safrole (B) isolated from the immature and mature fruit of $P$. auritum.

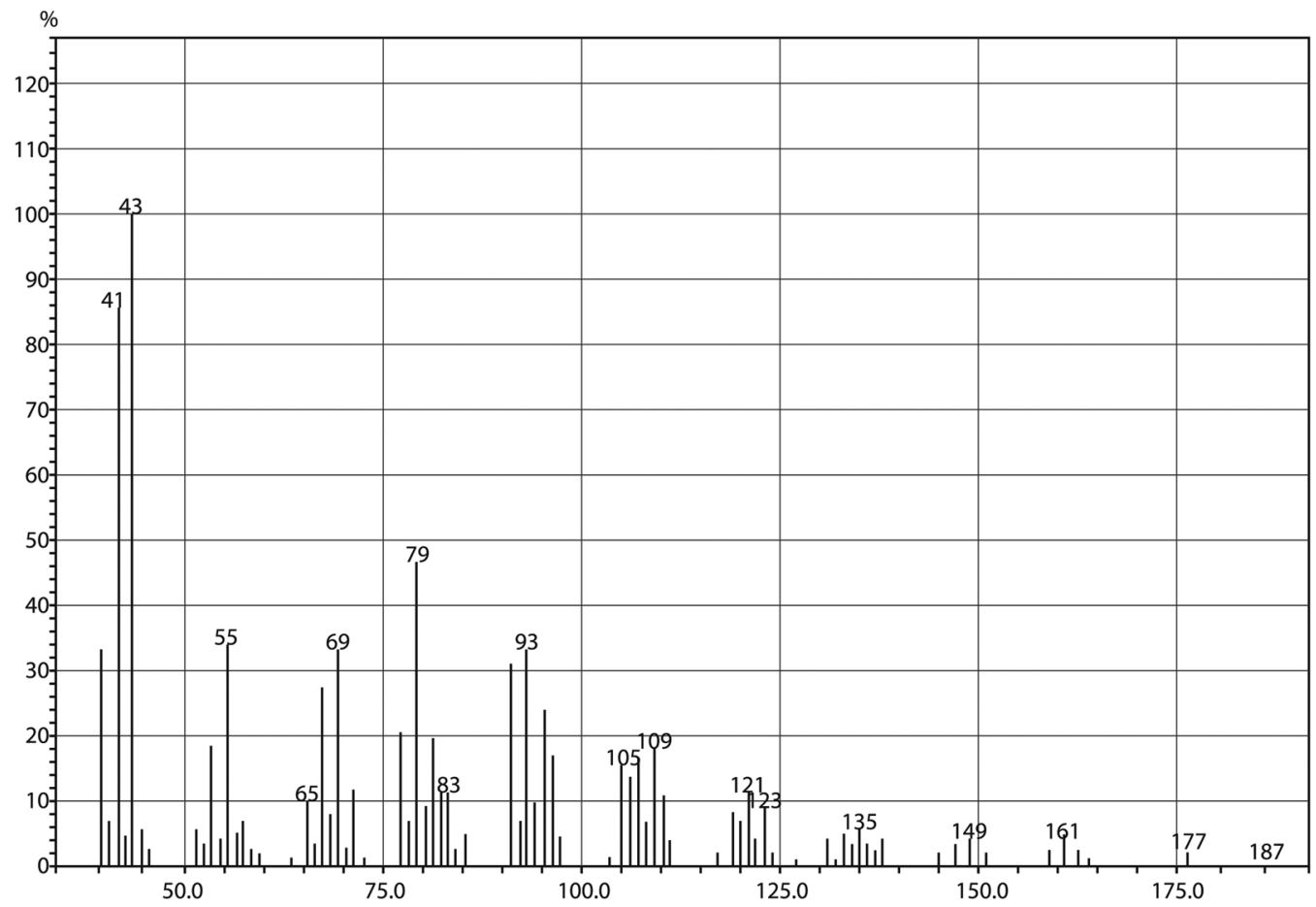

Fig. 2. Espectro de masas del trans-Z-alfa-bisaboleno epóxido.

Fig. 2. Mass spectrum of trans-Z-alpha-bisabolene epoxide. 
vectores de los géneros Phlebotomus y Lutzomia, que actúan como reservorios distintas especies de mamíferos y roedores (GonzálezLlanova et al., 2007). En la actualidad las opciones de tratamiento se reducen al uso de compuestos antimoniales pentavalentes, anfotericina $\mathrm{B}$, pentamidina y paromomicina, los cuales son compuestos potencialmente tóxicos, que pueden generar resistencia por parte del parásito, y los efectos secundarios en los pacientes pueden ser muy drásticos, por lo que la búsqueda de nuevos compuestos que presenten menor toxicidad, mayor efectividad y que generen menor resistencia es de suma importancia (WHO, 2010).

Actualmente, existen estudios realizados con los aceites esenciales de diversas plantas del género Piper como los mencionados en la revisión realizada por Pérez et al. (2012), donde se indica la actividad de los aceites esenciales de Piper aduncum contra Leishmania amazonensis; y se reporta además que la actividad de Piper auritum es similar al medicamento anfotericina. Otro estudio muestra que el aceite esencial de Piper hispidinervum tuvo también actividad contra Leishmania infantum, con un $\mathrm{CI}_{50}$ de $1.43 \mu \mathrm{g} / \mathrm{mL}$ (Bosquiroli et al., 2015).

Todo lo anterior demuestra la actividad contra la leishmania de los metabolitos secundarios aislados de diversas plantas de este género, que han sido estudiados, y se destaca la actividad de compuestos como los ácidos benzoicos, dillapioles, y adunchalconas (Bosquiroli et al., 2015). Otros autores reportan que el 2,6-dihidroxi-4-metoxichalcona aislado de la inflorescencia de Piper aduncum, presentó actividad contra Leishmania amazonensis a concentraciones de 0.5 a $24.0 \mu \mathrm{g} / \mathrm{mL}$ (Torres-Santos, Moreira, Kaplan, Meirelles, \& Rossi-Bergmann, 1999). Las hojas de Piper rusbyi también fueron trabajadas y se aisló la (+)-(R7,8S)-epoxi-5,6-didehidrokavaina y la chalcona flavokavaina que también presentaron actividad, con un $\mathrm{CI}_{50}$ de 81.9 y $11.2 \mathrm{mg} / \mathrm{mL}$, respectivamente (Flores et al., 2007).

En el presente estudio se logró aislar el trans-Z-alfa-bisaboleno epóxido, el cual se clasifica dentro de los sesquiterpenos y tuvo una buena actividad anti-Leishmania, con un $\mathrm{CI}_{50}$ de $50.0 \mu \mathrm{g} / \mathrm{mL}$. Según menciona Pérez et al. (2012) los aceites esenciales de ciertos tipos de plantas del género Piper son ricos en sesquiterpenos; esto se confirmó, con el tamizaje fitoquímico efectuado a la mezcla de frutos realizado en este estudio, donde se evidencia la presencia de este tipo de metabolitos secundarios.

En estudios más recientes se evaluó la actividad anti-Leishmania del aceite esencial del P.auritum, indicándose que dentro de los componentes más abundantes está el Safrol en un $86.91 \%$. El aceite esencial que contiene una mezcla de varios componentes tuvo un $\mathrm{CI}_{50}$ entre 12.8 y $63.3 \mathrm{mg} / \mathrm{mL}$ sobre los promastigotes y amastigotes en todas las especies de Leishmania utilizadas (Monzote et al., 2010).

A su vez se han realizado análisis por cromatografía de gases al aceite esencial de $P$. auritum y $P$. hispidinervum que revelaron la presencia del Safrol como principal componente; en concentraciones que varían del 60 al 80 $\%$ (Pretto et al., 2012; Sánchez, Pino, Correa, Naranjo, \& Iglesia, 2009; Pérez et al., 2012) junto a otros compuestos de menor concentración como lo son el cis-nerolidol en un $2.80 \%$, $\beta$-bisaboleno en $0.98 \%$, germacreno D en 3.11 $\%$, $\beta$-pineno en $1.45 \%$, entre otros muchos componentes ya identificados (Hernández et al., 2003). En la literatura se reportan diferencias entre la concentración y la composición de los metabolitos secundarios presentes en el aceite esencial, debidas principalmente a cambios en ciertos parámetros como el origen geográfico, etapa vegetativa de la planta, condiciones de almacenamiento, el método de extracción, entre otros (Pineda, Vizcaíno, García, Gil, \& Durango, 2012).

En los estudios anteriormente citados, no se indica cuál de los componentes presentes en el aceite esencial es el metabolito secundario responsable de la actividad antileishmaniásica; solo se menciona la presencia de ciertos metabolitos secundarios y se aclara que es el Safrol el metabolito presente en mayor concentración. Con este estudio se demuestra que el Safrol a pesar de ser el metabolito secundario presente en mayor concentración, no es el responsable 
de la actividad. Por el contrario, el trans-Zalfa-bisaboleno epóxido $\left(\mathrm{CI}_{50} 50.00 \mathrm{mg} / \mathrm{ml}\right)$ presentó una actividad más eficaz contra el parásito, que la mostrada por el antimoniato de meglumina, con un $\mathrm{CI}_{50}$ de $80 \mathrm{mg} / \mathrm{mL}$, el cual es catalogado como un fármaco de primera línea (Monzote et al., 2010).

El estudio y aislamiento de diversos compuestos como el reportado en este trabajo, que presenten una baja toxicidad y una alta eficiencia, son una opción viable para poder tratar diversas enfermedades. Es por esta razón que consideramos importante estudiar los compuestos aislados de plantas con potencial actividad contra la leishmaniasis; en tal sentido, el informe de las propiedades anti-parasíticas de este nuevo compuesto, representa un aporte en beneficio de la salud humana de los habitantes de regiones vulnerables a esta enfermedad.

\section{AGRADECIMIENTOS}

Este estudio fue patrocinado en parte por el Ministerio de Ciencia y Tecnología (MICIT), el Consejo Nacional para Investigaciones Científicas y Tecnológicas (CONICIT) por medio de los proyectos FI-291-09 y FI-490-2011, el Departamento de Investigación de la Universidad de Ciencias Médicas (UCIMED) y el Centro Regional de Occidente, Universidad de Costa Rica. Agradecimiento especial para Víctor Mora por su ayuda en la identificación de las plantas, a Laura Valerio encargada de la logística del proyecto y a los señores José Bolaños, Luis León y Hugo Pérez, por su labor asistencial y manejo de los roedores que se usaron en los experimentos. Los autores agradecen a Rodrigo Zeledón por el suministro de la cepa OCR de Leishmania sp. y también a un grupo de estudiantes de la UCIMED que colaboraron en el proyecto.

\section{RESUMEN}

La leishmaniasis es una enfermedad con una alta incidencia en el ser humano, que puede ser controlada, pero como los tratamientos tienen efectos secundarios importantes se han realizado estudios de diversas plantas con el fin de encontrar compuestos con actividad antileishmaniásica que presenten pocos efectos nocivos para el ser humano. El presente estudio consistió en realizar un tamizaje fitoquímico de la planta, para identificar la presencia de cumarinas, terpenos, triterpenos y azúcares reductores. El objetivo fue encontrar componentes químicos puros con actividad contra el parásito Leishmania sp. Por tal razón se purificaron los compuestos: trans-Z-alfabisaboleno y el Safrol, a los que se les realizaron pruebas del efecto anti parasitario que presentaron un $\mathrm{CI}_{50}$ de 50.0 $\mu \mathrm{g} / \mathrm{mL}$ y $0.0 \mu \mathrm{g} / \mathrm{mL}$, respectivamente. Además, se discute la importancia de estos nuevos hallazgos. El compuesto mayoritario presente en los aceites esenciales (Safrol) no es el componente que presentó la actividad. Es importante realizar estudios sobre su proyección en el tratamiento de la leishmaniasis.

Palabras clave: Leishmania; Leishmaniasis; Piper auritum; tamizaje fitoquímico; tratamiento.

\section{REFERENCIAS}

Barbosa, J. (2001). Antimoniato de meglumina. Revista da Sociedade Brasileira de Medicina Tropical, 34(1), 103-105.

Bezerra, G., Barros, E. J., \& De Francesco, E. (2014). Kidney involvement in leishmaniasis - a review. Brazilian Journal of Infectious Diseases, 18(4), 434-440.

Bosquiroli, L. S. S., Demarque, D. P., Rizk, Y, S., Cunha, M. C., Marques, M. C. S., Matos, M. F. C., ... Arruda, C. C. P. (2015). In vitro anti-Leishmania infantum activity of essential oil from Piper angustifolium. Revista Brasileira de Farmacognosia, 25(2), 124-128.

Carrillo, J., Chinchilla, M., Valverde, B., Porras, O., \& Mora, L. (1999). Visceral leishmaniasis in Costa Rica: first case report. Clinical Infectious Diseases: An official publication of the infectious diseases society of America, 29(3), 678-679.

Chinchilla-Carmona, M., Valerio-Campos, I. SánchezPorras, R., Bagnarello-Madrigal, V., Martínez-Esquivel, L., González-Paniagua, A., ... Rodríguez-Chaves, D. (2014). Actividad contra Leishmania sp. (Kinetoplastida: Trypanosomatidae) de plantas en una Reserva Biológica de Costa Rica. Revista de Biología Tropical, 62(3), 1229-1240.

Chinchilla-Carmona, M., Valerio-Campos, I., SánchezPorras, R., Mora-Chaves, V., Bagnarello-Madrigal, V., ... Venegas, J. C. (2011). Evaluación in vivo de la actividad antimalárica de 25 plantas provenientes de una Reserva de Conservación Biológica de Costa Rica. Revista Chilena de Historia Natural, 84, 115-123.

Chinchilla, M., Valerio, I., Sánchez, R., Mora, V., Bagnare1lo, V., Martínez, L., ... Apestegui A. (2011). In vitro 
antimalarial activity of extracts of some plants from a biological reverve in Costa Rica. Revista de Biología Tropical, 60(2), 881-891.

Croft, S., \& Coombs, G. (2003). Leishmaniasis - current chemotherapy and recent advances in the search for novel drugs. Trends in Parasitology, 19(11), 502-508.

De Castro, E., Cruz, I., Cañavate, C., de Melo, L. A., Sampaio, A. A., Madeira, F., ... Ferreira, C. M. (2015). Mixed infection of Leishmania infantum and Leishmania braziliensis in rodents from endemic urban area of the New World. BMC Veterinary Research, 11(71), 1-7.

Díaz, M. C., Bulus, G. D., \& Pica, Y. (2004). Métodos estadísticos para el análisis de resultados de toxicidad. In G. Castillo-Morales (Ed.), Ensayos toxicológicos y métodos de evaluación de calidad de aguas (pp. 99-124). México: IMTA.

Fernández, D., González-Baradat, B., Eleizalde, M., González-Marcano, E., Perrone, T., \& Mendoza, M. (2009). Trypanosoma evansi: A comparison of PCR and parasitological diagnostic tests in experimentally infected mice. Experimental Parasitology, 121(1), 1-7.

Flores, N., Cabrera, G., Jiménez, I. A., Piñero, J., Giménez, A., Bourdy, G., Cortes, F., \& Bazzocchi, I. (2007). Leishmanicidal constituents from the leaves of Piper rusbyi. Planta Médica, 73(3), 206-211.

García, A., Leyva, M. A., A. Martínez, J. R., \& Stashenko, E. E. (2007). Determinación de la composición química y actividad antioxidante in vitro del aceite esencial de Piper auritum Kunth (piperaceae) difundida en la costa colombiana. Scientia et Technica, 13(33), 439-442.

González-Llanova, B., Biosca-Echenique, G., Soto-Díaz, A., Naranjo-Díaz, M. J., Espadafor-López, B., \& García-Mellado, V. (2007). Leishmaniasis cutánea en paciente senegalés. Actas Dermo-Sifiliográficas, 98(1), 54-8.

Halkimi, W., Ahmad, F., Heng, K., \& Mohd, H. (2011). Chemical Compositions, Antioxidant and Antimicrobial Activities of Essential Oils of Piper caninum Blume. International Journal of Molecular Sciences, 12(11), 7720-7731.

Hasnain, M., Ghosh, P., Baker, J., \& Mondal, D. (2014). An evaluation of the performance of direct agglutination test on filter paper blood sample for the diagnosis of visceral leishmaniasis. The American Journal of Tropical Medicine and Hygiene, 91(2), 342-344.

Hernández-Torres, A., García-Vázquez, E., Bravo-Urbieta, J., Morell, E. B., Alcaraz-Vidal, B., Sánchez-Serrano, A., \& Gómez, J. (2015). La leishmaniasis visceral en la región de Murcia: estudio multicéntrico $1997-$ 2013. Infectio, 19(1), 24-30.

Hernández, L., Rodríguez, M., García, D., \& Pino, J. (2003). Actividad antidermatofítica in vitro de aceites esenciales. Revista cubana de plantas medicinales, 8(2), Recuperado en 24 de mayo de 2016, de http://scielo. sld.cu/scielo.php?script $=$ sci arttext\&pid $=\mathrm{S} 1028$ $47962003000200004 \& \operatorname{lng}=\mathrm{e} \overline{\mathrm{s} \& t \operatorname{lng}=\mathrm{es}}$

Jaramillo-Antillón, O., Espinoza-Aguirre, A. \& LoboPhilp, R. (2009). Estado actual de la leishmaniosis en Costa Rica. Acta Médica Costarricense, 51(3), 158-164.

Jiménez, A. E., Rojas, J. C., Vargas, F., \& Herrero, M. V. (2000). Temporal and spatial variation of phlebotomine (Diptera: Psychodidae) community diversity in a cutaneous leishmaniasis endemic area of Costa Rica. Journal of Medical Entomology, 37(2), 216-221.

Leyva, M., Marquetti, M. C., Tacorente, J. E., Scull, R., Tiomno, O., Mesa, A., \& Montada, D. (2009). Actividad larvicidad de aceites esenciales de plantas contra Aedes aegypti (L.) (Diptera: Culicidae). Revista Biomédica, 20, 5-13.

Luize, P. S., Tiuman, T. S., Morello, L. G., Korehiza, P., Ueda-Nakamura, T., Dias-Filho, B. P., ... Nakamura, C. V. (2005). Effects of medicinal plant extracts on growth of Leishmania (L.) amazonensis and Trypanosoma cruzi. Revista Brasileña de Ciencias Farmacéuticas, 41(1), 85-94.

Monzote, L., García, M., Montalvo, A. M., Scull, R., \& Miranda, M. (2010). Chemistry cytotoxicity and antileishmanial activity of the essential oil from Piper auritum. Memórias do Instituto Oswaldo Cruz, 105(2), 168-173.

Pérez, G., Ramos-López, M. A., Sánchez-Miranda, E., Fresán-Orozco, M. C., \& Pérez-Ramos, J. (2012). Antiprotozoa activity of some essential oils. Journal of Medicinal Plants Research, 6(15), 2901-2908.

Pineda, R., Vizcaíno, S., García, C. M., Gil, J. H., \& Durango, D. L. (2012). Chemical composition and antifungal activity of Piper auritum Kunth and Piper holtonii C. DC. against phytopathogenic fungi. Chilean Journal of agriculturalresearch, 72(4), 507-515.

Pretto, I., Evaldt, G., Machado, A., Cibulski, S. P., Roehe, P. M., Alves, L. A., Brittes, M., Figueiró, R. M., Cassel, E., \& Lino, G. (2012). Chemical composition and amoebicidal activity of Piper hispidinervum (Piperaceae) essential oil. Industrial Crops and Products, 40, 292-295.

Sánchez, R. (2000). Reserva Biológica Alberto Manuel Brenes. San José, Costa Rica: Ministerio de Ambiente y Energía. 
Sánchez, Y., Pino, O., Correa, T. M., Naranjo, E., \& Iglesia, A. (2009). Estudio químico y microbiológico del aceite esencial de Piper auritum Kunth (Caisimón de anís). Revista de Protección Vegetal, 24(1), 39-46.

Sharapin, N. (2000). Fundamentos de Tecnología de productos fitoterapéuticos (1 Ed.). Colombia: Serie Ciencia y Tecnología.

Torres-Santos, E. C. T., Moreira, D. L., Kaplan, M. A., Meirelles, M. N., \& Rossi-Bergmann, B. (1999). Selective effect of 2',6'-dihydroxy-4'methoxychalcone isolated from Piper aduncum on Leishmania amazonensis. Antimicrobial Agents and Chemotherapy, 43(5), 1234-1241.
WHO. (2010). Control of the Leishmaniasis: Report of a Meeting of the WHO Expert Committee on the Control of Leishmaniases, WHO Technical Report Series. 186-9491.

WHO. (2016). Leishmaniasis in high-burden countries: an epidemiological update based on data reported in 2014. Weekly epidemiological record, 91(22), 285-296.

Wink, M. (2012). Medicinal Plants: A Source of AntiParasitic Secondary Metabolites. Molecules, 17(11), 12771-12791. 